Aus dem Orthopädischen Institut der Universität in Freiburg i. B. (Direktor: Prof. Ritschl.)

\title{
Osteopsathyrosis idiopathica.
}

\section{Von Dr. J. Lewy in Freiburg-Günterstal.}

Mit dem Ausdruck „Os te opsathyros is" bezeichnet man ganz allgemein eine über das nornale Maß hinaus gesteigerte Knochenbrüchigkeit ohne Rücksicht auf die ihr zugrunde liegenden Ursachen. Diese können mannigfaltiger Natur sein. Gesteigerte Knochenbrüchigkeit findet sich bei der Rachitis, der Osteomalazie, bei atrophischen Zuständen der Knochen, wie sie sich im Anschluß an Kachexie, im Greisenalter, als Begleiterscheinung verschiedener zentral-nervöser Erkrankungen (Tabes, progressive Paralyse - neurotische Atrophie) herausbilden, bei Tumoren und Zysten in Knochen, Lues etc.

Die Osteopsathyrosis idiopathica, von der im Nachstehenden die Rede sein soll, stellt, wie schon der Name besagt, ein Krankheitsbild für sich dar, dessen Eigenart, wenn auch noch nicht erschöpfend klargelegt, so doch in der letzten Zeit durch histologische und röntgenologische Untersuchungen in etwas helleres Licht gerückt worden ist.

Das wesentlichste Symptom des eigenartigen, nicht gerade häufig beobachteten Krankheitszustandes ist die nicht selten schon intrauterin in die Erscheinung tretende, überaus gesteigerte $\mathrm{Brüchigkeit}$ der Knochen, sowohl der langen Röhren-, wie der kurzen, platten Knochen. Daß dabei die ersteren in wesentlich höherem Prozentsatz an der Frakturierung beteiligt sind, entspricht nur den natürlich gegebenen Verhältnissen.

Ein ganz geringfügiges Trauma, ein leichter Fall oder Stoß, selbst schon eine gesteigerte Muskelkontraktion reicht aus, um nicht nur Infraktionen herbeizuführen, sondern eine Diaphyse völlig quer zu durchtrennell. Oft genug kommen die Brüche, da sie meist nur mit geringem Schmerz und mäßiger Blutung einhergehen, garnicht oder erst verspätet zur Kenntnis.

Die bei weitem größte Zahl der bisher beobach teten Fälle betraf Kinder in den ersten Lebensjahren bis zum Pubertätsalter bzw. beendeten Wachstum, meist Knaben. Ueber das Wachstumsalter hinaus scheint sich die Veranlagung, bei der ein Erblichkeitscharakter unverkennbar ist - de Cortes ${ }^{1}$ ) konnte die Veranlagung in seinem Falle bis in die vierte Generation zurück verfolgen - zu verlieren. Es sind nur vereinzelte Fälle bekannt geworden, in denen die Veranlagung noch in vorgeschrittenem Alter persistierte. So berichtet Biggs ${ }^{2}$ ) über einen Fall, in welchem die Krankheit erst mit dem 20. Lebensjahr einsetzte und mit dem 30 . verschwand. Während dieses zehnjäh rigen Zeitraumes ereigneten sich 22 Frakturen, welche die Oberarme und Oberschenkel betrafen.

1) Zeitschrift für orthopädische Chirurgie, Bd, 29, H. 3 u. 4 . a) Pennsylv. med: Bull. 1903, No. 12. 
Die Anzahl der Brüche bei einem einzelnen Individuum kann enorm groß sein.

Matsuoka ${ }^{1}$ ) zählte bei einem Schwesternpaar innerhalb zwei Jahren 18 Frakturen, Lovett und Nichols ${ }^{2}$ ) bei einem Kinde bis zum zehnten Lebensmonat bereits zehn Knochenbrüche, Schmidt ${ }^{3}$ ) in sieben Fällen nicht weniger als 130 Frakturen, darunter zwei Patienten mit je 40 Brüchen, Langmend ${ }^{4}$ ) konstatierte durch Radiographie bei einem neugeborenen Kinde schon elf Frakturen der Extremitätenknochen.

Im allgemeinen pflegen die Brüche, deren bei weitem größte Zahl die Diaphysen betrifft, schnell zur Heilung zu gelangen, teils unter normaler, teils unter geringer Kallusbildung. Oefters beobachtet ist ein symmetrisches Befallenwerden der oberen oder unteren Extremitäten. Neben den Frakturen kommt es zu Infraktionen, Verbiegungen und Verkrümmungen, die oft zu geradezu grotesken Formen führen.

Ueber die Ursache der Affektion ist viel gestritten worden, ohne daß man bis heute zu einer Einigung oder einwandfreien Erklärung gelangt wäre.

Hochsinge ${ }^{5}$ ) ist der Meinung, da $B$ es sich um eine generelle fötale Knochenerkrankung handelt, für die eine entzündliche Schädigung anzuschuldigen ist. $\mathrm{Schmidt}^{3}$ ) glaubt, daß ein rachitischer Proze $B$ in Frage kommt, der mit Störungen trophischer Nerven verbunden ist. Auch Peiser ${ }^{6}$ ) nimmt eine Trophoneurose an. Dillenburger ${ }^{7}$ ), der in seinem Falle eine Pachymeningitis unbekannter Ursache beobachtete, rechnet mit der Möglichkeit, daß dieser Befund einen neuen Anhalts. punkt für die Erforschung der Aetiologie geben könne, die das Zentralnervensystem bisher noch zu wenig beachtet hat. Uebrigens hatte schon Morea ${ }^{8}$ ) unter dem Eindruck der Entdeckungen von Charcot über den Einfluß des Nervensystems auf die Knochenernährung im Anschluß an eine einschlägige Beobachtung die Hypothese aufgestellt, daß die Ursache der Osteopsathyrosis in einer Knochenatrophie infolge Läsion der Vorderhörner des Rückenmarkes zu suchen sei. Mit den von anderen Autoren für die Erklärnng der Osteopsathyrosis herangezogenen Begriffen, wie „Mißverhältnis zwischen anorganischem und organischem Teil“, "Kundgebung der alterierten Qualität der organischen Substanz", „Insuffizienz der organischen Stoffe“, „Alteration der chemischen Zusammensetzung" läßt sich füglich garnichts anfangen.

Was auch immer die eigentliche Ursache der Erkrankung bilden mag, keinesfalls darf das Krankheitsbild mit der infantilen Osteomalazie oder der fötalen Rachitis zusammengeworfen werden, da die klinischen wie histologischen und röntgenologischen Untersuchungsbefunde dafür zu sehr differieren.

Was die histologischen Veränderungen betrifft, so ist folgendes beobachtet: Hochgradiger Schwund der Spongiosa und Compacta (Osteoporosis). Nach Looser ${ }^{9}$ ), der die Bezeichnung Osteopsathyrosis idiopathica verwirft und das Krankheitsbild für identisch hält mit dem der Osteogenesis imperfecta, eine Anschauung, der auch Nathan ${ }^{10}$ ) huldigt, besteht das Wesen der Krankheit in einer mangelhaften Funktion der endostalen und periostalen Osteoblasten bei normaler Bildung derselben. Daher ist die Knochenapposition mangelhaft, während die Resorption normal ist. Die Folgen der mangelhaften Knochenapposition sind hochoradige Atrophie und mangelhaftes Dickenwachstum. Am feineren Bau der Knochensubstanz zeigt sich die mangelhafte Apposition am großen Zellreichtum der Bälkchen und an der körnig-krümeligen Verkalkung der Knochengrundsubstanz. Die Epiphysenknorpel sind zunächst normal und zeigen erst später regressive Veränderungen infolge von Raumbeengung der normal in die Breite wachsenden Epiphysenscheibe durch die mangelhaft wachsende, sie einschließende Knochenschale. Das Knochenmark ist normal, nur an Stellen mechaniseher Reizung fibrös umgewandelt.

Lovett und Nichols (l. c) beschreiben die wesentliche Abweichung in der Knochenneubildung sowohl an der Epiphysenlinie, wie am Periost des cenaueren: Die Epiphyse besteht aus normalem, hyalinem Knorpel mit Knorpelzellen, die, wie in der normalen Epiphyse, diaphysenwärts sich in Säulenreihen anordnen, umgeben von bindegewebiger Matrix. An der Diaphysengrenze nun bleiben diese Knorpelzellen erhalten, ihre Kapseln bersten nicht. Massen von diesen Zellen mit der dazwischen liegenden Matrix ragen in die Diaphyse hinein und stellen den Anfang neuer Trabekeln dar, während im normalen Knochen die verkalkte Matrix allein bestehen bleibt. Die nicht geborstenen Knorpelzellenkapseln verdicken sich durch Kalkablagerungen und bilden gemeinsam

(1) Zeitschrift für Chirurgie Bd. 98, H. 4 u. 5. - ${ }^{2}$ ) Brit. med. journ. 13. Oktober 1906. - 3) Inauguraldissertation, Leipzig 1901. - ${ }^{4}$ ) Brit. med. journ. 9. November 1907. - 5) Wiener medizinische Wochenschrift 1909, No. 14. - ' ') Münchener "medizinische Wochenschrift 1907, No. 45. - ?) Inauguraldissertation Bonn 1908. - ${ }^{8}$ ) Thèse de Paris. 1894. $\because 9$ ) Grenzgebiete der Medizin und Chirurgie Bd. 25, H. 1 u. 2. - 10) Amer. journ. of the med. soiences. Januar 1905 . mit den benachbarten Zellen die neuen Trabekeln, während normalerweise die Ossifikation erzeugt wird durch Osteoblastentätigkeit innerhalb der Matrix ohne die hier bestehen bleibenden Knorpelzellen. Ferner bilden die neuen Knochenzellen keine Ausläufer; sie bleiben groß, oval, entwickeln keine Kanälchen und sind viel zahlreicher als im normalen Knochen. Das Mark, sowohl epiphysealen wie periostalen Ursprungs, ist ein ödematöses, myxödematöses, zusammenhängendes Gewebe.

Hildebrand ${ }^{1}$ ) hebt in seinem mikroskopisehen Befund nament lich hervor: mangelhafte Ausbildung der Knochensubstanz, besonder die periostale Knochenbildung ist sehr gering; zahlreiche Nekrosen des Knochengewebes; Erhaltensein großer Inseln von Knorpelgrundsubstanz in der Spongiosa; Epiphysenknorpel normal zum Unterschied von der Chondrodystrophia foetalis.

Nekrose der Knochenbälkchen mit entzündlicher Veränderung des Knochenmarkes in der Umgebung, sowie fibröse Umwandlung des Markes hat auch Döring ${ }^{2}$ ) gesehen.

Müller ${ }^{3}$ ) betont das meist völlige Fehlen von periostalem Knochen, dagegen manchmal vermehrte endochondrale Knochenbildung. Ueberall aber fehlte das für Rachitis charakteristische Verhalten der Knorpelzellen in den Epiphysenlinien, sowie die Unregelmäßigkeit der hyperplastischen Zone und der Verknöcherungsschicht. is . . $k^{*}$

Nach Michel ${ }^{4}$ ) sind die präparatorischen Vorgänge am Knorpel ungefähr normal. Hingegen fehlte so gut wie ganz die endochondrale Ossifikation. Die Knochen bestehen aus einem äußerst dünnen Mantel verkalkter Knochenbälkchen, wodurch sich die hochgradige Knochenbrüchigkeit vollauf erklärt.

Schuchardt ${ }^{5}$ ) war der Ansicht, daß die geringe Dickenaus. bildung der Knochen durch eine Funktionsversagung des Periosts bzw. der durch das Periost zu leistenden Knochenapposition zu erklären sei.

Dem widersprechen die Befunde Dörings ${ }^{6}$ ), in dessen Fall aus der Göttinger Klinik das Periost guten Kernreichtum, ja Kernvermehrung zeigte. Döring hebt besonders das Vorhandensein scholliger Gebilde im Knochenmark, inmitten der großen, vielgestaltigen Markräume, hervor. Diese gehen zum Teil direkt in normale Knochenbälkchen über. Nekrotische Knochenpartien führt dieser Autor auf Traumen (Dehnung, Kompression) zurück. Was das biologische Verhalten des Periosts anbetrifft, so sei an dessen Produktionsfähigkeit nicht zu zweifeln dagegen möge es eine die Norm überschreitende Resorptionskraft besitzen, deren Folge eine Dysplasie bzw. Atrophie der Corticalis sei.

Auch Scheib ${ }^{7}$ ) fand die Knorpelanlage, die Wucherungsschich und präparatorische Verkalkungszone normal; dagegen bestand durchweg das Bild einer Hemmung in der Osteogenese und ferner einer gesteigerten Resorption der spärlich angelegten Knochenbälkchen. Bezüglich der Pathogenese nimmt Scheib an, daß das blutarme, fibrös Knochenmark Osteoblasten anbildet, welche, in ihrer Struktur verändert, ihre Funktion eingebüßt haben und sich in Knochen zerstörende Elemente (Osteoklasten) umwandeln. funde.

Soviel über die wichtigsten, bisher erhobenen histologischen Be-

Was nun die Ergebnisse der rön tgen ologis chen Un tersuchungen betrifft, so hat neuerdings Rudolf Maier ${ }^{8}$ ) in einer aus der Joachimthalschen Universitätspoliklinik hervorgegangenen Arbeit einen Ueberblick über die röntgenologischen Grundlagen der idiopathischen Osteopsathyrose gegegeben und gleichzeitig die Ergebnisse bei den verwandten Krankheitsformen, der Osteogenesis imperfecta und der infantilen Osteomalazie, zum Vergleich herangezogen. An Hand seiner Angaben ist die nachstehende Uebersichtstabelle zusammengestellt, die auch die Rachitis berücksichtigt.

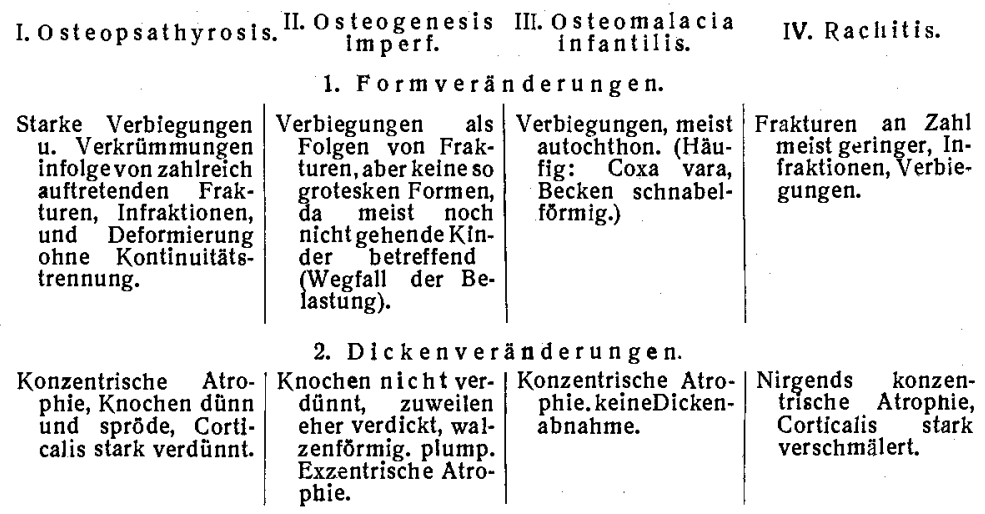

1) Virchows Archiv 1899, Bd. 158, H. 3. $-{ }^{2}$ ) Diese Wochenschrift 1904, No. 48. $-{ }^{3}$ ) Inauguraldissertation, München 1893. De $77, \mathrm{~S}$. 7) Beiträge zur klinischen Chirurgie Bd. 26, S. 93. - ${ }^{8}$ ) Zeitschrift für 7) Beiträge zur klinischen Chirurgie Bd. 26 ,
orthopädische Chirurgie Bd. 27, H. 1 u. 2. 
3. Strukturveränderungen.

\begin{tabular}{|c|c|c|c|}
\hline $\begin{array}{l}\text { 3ald deutlich, bald un- } \\
\text { deutlich, bald nur } \\
\text { diffuse Schatten. } \\
\text { Quere, weiBe Linien in } \\
\text { der Diaphyse. } \\
\text { (frü here Frakturen?) }\end{array}$ & $\begin{array}{l}\text { Compacta nur andeu- } \\
\text { tungsweise vor- } \\
\text { handen. Spongiosa } \\
\text { unregeimäBig. }\end{array}$ & $\left|\begin{array}{r}\text { Struktur deutlich, je- } \\
\text { doch Osteoporose. }\end{array}\right|$ & Starke Porose. \\
\hline $\begin{array}{c}\text { tarke Durchgängig- } \\
\text { keit. }\end{array}$ & $\begin{array}{l}\text { 4. Strahlendur } \\
\text { Stellenweise ver- } \\
\text { mehrt. }\end{array}$ & $\begin{array}{l}\text { c h } 1 \text { a s s i g k e i t. } \\
\text { Erhöht. }\end{array}$ & $\begin{array}{c}\text { Starke Durchlässig. } \\
\text { keit. }\end{array}$ \\
\hline $\begin{array}{l}\text { Ausgedehnter, zuwei- } \\
\text { len geringer, auch } \\
\text { fehlender Kallus. } \\
\text { Manchmal geringe } \\
\text { Diastase der Bruch- } \\
\text { enden. }\end{array}$ & $\begin{array}{l}\text { 5. Frak } \\
\text { Große Diastase mit } \\
\text { wenig schatten- } \\
\text { gebendem Kallus, } \\
\text { derbald stark, bald } \\
\text { dünn ist, auch } \\
\text { fehit. }\end{array}$ & $\begin{array}{l}\text { Geringe oder keine } \\
\text { Kallusbildung } \\
\text { ohne jeden Kalk- } \\
\text { gehalt. }\end{array}$ & $\begin{array}{l}\text { Kallus meist gering } \\
\text { oder fehlt. }\end{array}$ \\
\hline $\begin{array}{l}\text { Keine Vergrößerung, } \\
\text { zuweilen Abplat- } \\
\text { tung, abnorm. Durch- } \\
\text { lassigkeit und Struk- } \\
\text { tur, wie bei den Dia- } \\
\text { physen. }\end{array}$ & Normal. & $\begin{array}{l}\text { hy y e n. } \\
\text { Außer Osteoporose } \\
\text { und starker Durch- } \\
\text { leuchtbarkeit keine } \\
\text { gröberen Verănde- } \\
\text { rungen. }\end{array}$ & $\begin{array}{l}\text { Auftreibung der Epi } \\
\text { physen. }\end{array}$ \\
\hline
\end{tabular}
tur, wie bei den Dia-

7. Ep iphysenfugen.

Normal, höchstens zu- $\mid$ Meist geradlinig, kei- $\mid$ Zuweilen stark ver- Verbreitert, unregel-

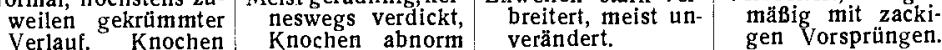
nicht verkîrzt. kurz.

Nach diesem kurzen Ueberblick über die Literatur der Osteopsathyrosis idiopathica, der auf Vollständigkeit keinen Anspruch macht, möge die Beschreibung eines einschlägigen Falles folgen, der uns längere Zeit beschäftigt hat.

Clara H., ein jetzt $5 \frac{1}{2}$ Jahre altes, im allgemeinen gesundes Mädchen, ist das fünfte von sechs Geschwistern. Die Eltern sind gesund. Nach ihren Berichten scheint das Kind schon intrauterin Frakturen der Oberschenkel erlitten zu haben. Die Geburt soll normal vonstatten gegangen sein, doch wären beide Oberschenkel stark geschwollen und wie gebrochen gewesen. Die ersten Zähne bekam C. mit neun Monaten. Noch vor Vollendung des zweiten Lebensjahres trat aufs neue bei den ersten Gehversuchen eine Fraktur des linken Oberschenkels ein. Im Laufe der Zeit kam es dann aus ganz geringfügigen Veranlassungen, wie Umfallen, Anstoßen, zu erneuten Brüchen, die noch fünfmal die Oberschenkel und viermal die Unterschenkel betrafen. Die oberen Extremitäten und sonstige Knochen blieben verschont. Von den Geschwistern sind die beiden ältesten und das jüngste völlig gesund. Ein jetzt 13 jähriger Bruder jedoch, sowie eine jetzt 10 jährige Schwester haben ebenfalls, als sie das Gehen lernten, beide zweimal den Oberschenkel gebrochen. Weitere Brüche sind bei ihnen indessen nicht mehr vorgekommen. Wie aus den beigefügten Röntgenbildern ersichtlich ist, haben sich die Frakturen

Fig. 1.

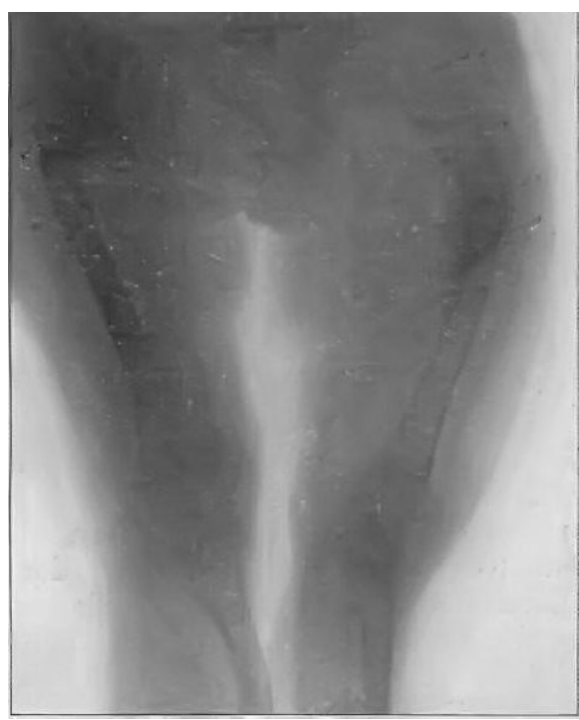

der gestörten statischen Verhältnisse gehäufte Anbildung von Knochensubstanz. Die Struktur der Knochen im allgemeinen ist undeutlich, auch abgesehen von dem Schuldkonto der Reproduktionen, die ja in
Fig. 2.

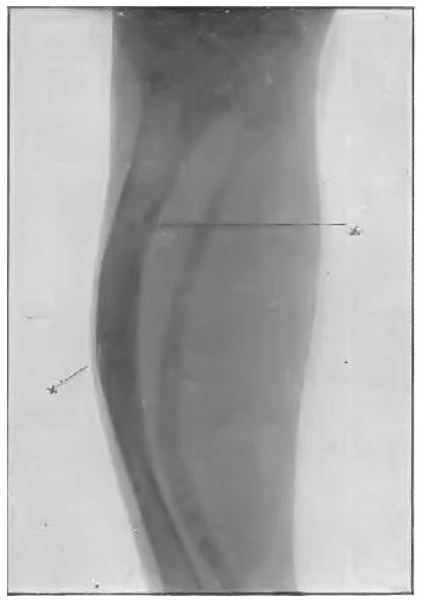

Fig. 3.

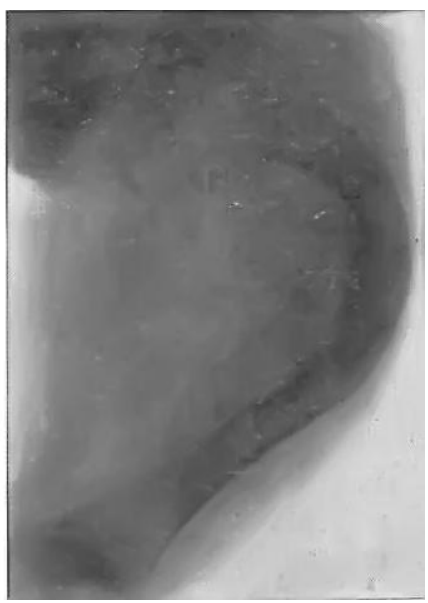

technischer Beziehung noch manches zu wünschen übrig lassen; bemerkenswert ist die Dünnheit der Corticalis. Epiphysen und Epiphysenfugen weisen nichts Besonderes auf.

Zur Besserung der statischen Verhältnisse entschlossen wir nus zur Ausführung der Keil-Osteotomie an der Tibia. Die histologische Untersuchung des entfernten Knochenstückes (Pathologisches Institut der Universität Freiburg) ergab folgendes: „Makroskopisch eine Verdünnung der Corticalis. Mikroskopisch sind die Knochenbälkchen gut ausgebildet und von normaler Dicke; irgendwelche krankhaften Veränderungen sind an ihnen mit Sicherheit nicht nachweisbar. Osteoblasten sind in genügender Menge vorhanden, doch erscheinen ihre Reihen ziemlich niedrig. Auffallend ist, daß das rote Knochenmark vollkommen fehlt und an seine Stelle ein fibröses Gewebe getreten ist."

Was den Heilverlauf anbetrifft, so ist ein trotz aller Vorsicht eingetretener Zwischenfall charakteristisch für die unzureichende Regenerationskraft bzw. starke Brüchigkeit der Knochen, mit der man es hier zu tun hat. Als 8 Wochen nach dem Eingriff, also zu einem Zeitpunkt, wo man schon eine leidliche Konsolidation erwarten konnte, der behutsame Versuch gemacht wurde, Bewegungen im Kniegelenk auszuführen, um eine Versteifung dieses Gelenkes zu vermeiden, erfolgte unter deutlichem Knacken eine erneute Infraktion an der Operationsstelle. Es scheint demnach doch nicht immer zutreffend zu sein, daß die Heilung der Frakturen sich ebenso leicht vollzieht wie bei sonstigen Brüchen.

Wir sind auch etwas skeptisch in bezug auf die Frage geworden, ob es zweckmäßig ist, so schnell mit der Osteotomie bei der Hand zu sein zu einem Zeitpunkt, wo der Krankheitsprozeß noch nicht zum Abschluß gekommen ist, bzw. die vorhandene Disposition noch mit der Wahrscheinlichkeit erneuter Brüche rechnen läßt. Dann kommt es, wie in unserem Falle, ohnedies noch zuweilen bei Gelegenheit neuer Brüche dazu, daß die Natur selbst eine Korrektur der gestörten statischen Verhältnisse vornimmt. Mit besseren und dauernden Erfolgen wird man rechnen können, wenn man gegebenenfalls erst zu einer späteren Zeit, in der einige Aussicht auf stabile Verhältnisse besteht, zur Operation schreitet.

Diesen Zeitpunkt möglichst bald herbeizuführen, mit anderen. Worten gegen die Veranlagung bzw. die abnorme Verfassung der. Knochen mit innerlich zu verabreichenden Mitteln anzukämpfen, bietet vor der Hand leider noch wenig Aussicht auf Erfolg. Wir haben unsere Kranke längere Zeit hindurch Thyreoidintabletten nehmen lassen, da die Schilddrüsentherapie von mehreren Seiten empfohlen worden ist. Von einem Erfolge können wir nicht berichten. Der Gedanke, auf dem Wege der Beeinflussung der inneren Sekretion auf die Krankheitsursache Einfluß zu gewinnen, liegt gewiß nahe. Sind doch mancherlei Beziehungen zwischen Knochen und Drüsen mit innerer Sekretion bekannt. Ich erinnere an das Krankheitsbild der Osteomalazie der Schwangeren, bei denen die Funktion der Ovarien ruht, an die Akromegalie infolge einer Ueberfunktion der Hypophysis cerebri bei Adenom- entsprechender Abduktion des Oberschenkels. Bemerkenswert ist metrische Auftreten der Frakturen: in bezug auf Zahl und Lokalisation der Brüche liegen auf beiden Seiten annähernd die gleichen Verhältnisse vor

Die enormen Verkrümmungen sind deutlicher in den beiden nächsten Abbildungen sichtbar.

Von den vier Frakturen, welche die Unterschenkel betroffen haben sollen, ist mit Deutlichkeit im Bilde nur noch eine nachweisbar. Der vermehrte Schatten im Bereich der Markhöhle, auf den in Fig. 3 oberhalb der Frakturstelle hingewiesen ist, dürfte wohl zu erklären sein durch die Bildung von Spongiosa, wie sie teils angeregt wurde durch die Frakturheilung (Markkallus), teils aufzufassen ist als eine zum Ausgleich 
bildung in dieser, an das Zurückbleiben der Entwicklung bei Ausfall der Thymusfunktion, an das Offenbleiben der Epiphysenfugen infolge Unterfunktion der Genitalorgane, das zum echten Gigantismus führen kann, an den Kretinismus infolge Fehlens oder Unterwertigkeit der Schilddrüsensekretion, Zusammenhänge, auf die neuerdings Fleischmann ${ }^{1}$ ) wieder die Aufmerksamkeit gelenkt hat. Vielleicht gelingt es einmal, wenn die auf diese Fragen gerichteten Untersuchungen Ergebnisse gezeitigt haben, die der Kritik standhalten können, auf diesem Wege dem eigenartigen Leiden beizukommen. Vor der Hand erscheint uns die Empfehlung der Schilddrüsen - oder auch Adrenalintherapie - noch etwas zu mystischen Charakter zu haben.

So bleibt nichts anderes übrig, als die an idiopathischer Osteopsathyrose leidenden Kinder, man möchte sagen in Watte zu packen, d. h. sie denkbar vor äußeren mechanischen Insulten zu behüten, ihren allgemeinen Kräftezustand tunlichst zu heben und nach Möglichkeit dafür Sorge zu tragen, daß die Frakturen korrekt zur Heilung gelangen und der Störung der statischen Verhältnisse infolge der Verbiegungen und Verkrümmungen durch rechtzeitig ergriffene Maßnahmen in geeigneter Weise entgegengetreten wird. 